\title{
Utilización de cuadernos didácticos para educar en valores: un reto en las Escuelas de Baloncesto de la Fundación Realmadrid
}

\author{
Using workbooks to teach values: a challenge in \\ fundación Realmadrid basketball schools
}

\author{
Gema Ortega, Ana Concepción Jiménez, Javier Giménez, Pedro Jiménez y Jorge Franco
}

\begin{abstract}
PALABRAS CLAVE: Baloncesto, valores educativos, materiales didácticos. RESUMEN: La enseńanza y aprendizaje del baloncesto en las etapas de iniciación supone un doble reto para los entrenadores/as, dada la posibilidad de contribuir al incremento de los valores educativos de los deportistas, simultáneamente a su labor específica del baloncesto. La presencia de programas concretos, que ayuden a los entrenadores/as a transmitir valores educativos, puede ser muy importante, siempre y cuando sean programas asequibles y útiles en el ámbito del entrenamiento y la competición. En las Escuelas de Baloncesto de la Fundación Realmadrid, en el marco del proyecto denominado "Por una Educación REAL: Valores y Deporte", se ha creado un programa llamado "La Alineación de los Valores" con el fin de facilitar la labor educativa de los participantes. Se han elaborado unos cuadernos didácticos para uso de los entrenadores/as, los jugadores/as y sus familias. En la temporada 2011-12, los entrenadores/as que han utilizado los cuadernos didácticos como herramientas de trabajo, con diferentes equipos de categoría benjamín, alevín, infantil y cadete, han sido 17. Los resultados son positivos, puesto que: a) estos materiales didácticos han permitido incluir y estructurar adecuadamente determinados valores educativos en la programación táctica y técnica de los entrenadores/as durante la temporada; b) ha fomentado el diálogo y la reflexión de los participantes, tanto en los entrenamientos como en la competición; c) la puesta en práctica ha deriva-
\end{abstract}

do a propuestas susceptibles de mejora para próximas temporadas. KEY WORDS: Basketball, educative values, materials.

ABSTRACT: The teaching and learning of basketball in the stages of initiation presents a dual challenge for coaches given the opportunity to contribute to increasing the educational value of sports, simultaneously with its specific work of basketball. The presence of specific programs to help coaches / as to transmit educational values can be very important, provided they are affordable and useful programs in the field of training and competition. In Fundación Realmadrid Basketball Schools, under the project entitled "For a Real Education: Values and Sport" has created a program called "Line up of Values" in order to facilitate the educational efforts of the participants. It has been designed workbooks for use by basketball teachers, the children and their families. In the 2011-12 season, teacher notebook has been used as a tool by seventeen teachers, with different youth teams: under-10, under- 12 , under- 14 and under- 16 . The results are positive, since: a) these materials have led to include and to structure certain educational values in the teacher's tactical and technical programming during the season, b) has promoted dialogue and reflection by participants, both in training and competition c) implementation has led to proposals for improvement for next season.

\section{Introducción}

¿La enseñanza del deporte, sobre todo en las etapas de iniciación y formación, puede transmitir valores educativos? La mayoría de los profesionales que nos dedicamos a la enseñanza del deporte consideramos que no solo se puede, sino que se debe transmitir aquellos valores educativos que el propio deporte invita a enseñar. Existen determinados programas para la adquisición y mejora de los valores educativos a través de la práctica deportiva. Son herramientas, en muchos casos, de cuidada elaboración realizadas por especialistas en la materia (Hellison, 1995; Comunidad de Madrid, 2005; FAD, 2009; Fundación Cultura y Deporte, 2012; Fundación FEB, 2012).

Dirección para correspondencia: Dña. Gema Ortega. Fundación Realmadrid. C.C. La Esquina del Bernabéu. C/ Padre Damián, 28036 Madrid.

E-mail: gortega@realmadrid.es

Este trabajo, enmarcado en el proyecto "Por una educación REAL: Valores y Deporte", ha sido financiado por la Fundación Realmadrid (2008-09/ 2011-12).
En baloncesto, numerosas escuelas de iniciación y formación tienen como objetivos la educación en valores. Abogan porque sus entrenadores/as fomenten una actitud de respeto, compañerismo, autonomía, e igualdad a sus deportistas. Por este motivo, entendemos que la planificación de dichos valores, a corto y medio plazo, debería estar presente en las programaciones para intervenir no solo cuando se presenta una situación idónea, sino para fomentarlo intencionadamente en el transcurso de los entrenamientos y la competición.

La Fundación Realmadrid ha realizado diferentes estudios con los entrenadores/as de sus Escuelas de Baloncesto, evaluando qué y cómo transmiten valores a los jugadores/as y cuáles son las dificultades encontradas en el día a día. Partiendo de las necesidades formativas de los entrenadores/as, y habiendo revisado diferentes estudios (Consejo Superior de Deportes, 2004; Fraile et al. 2008; Gutiérrez, 2006; Jiménez, 2000, 2008; Jiménez y Durán, 2006; Lleixá y Soler, 2004; Mosquera, 2004), ha creado un proyecto educativo a través 
de la enseñanza del baloncesto y del fútbol, denominado "Por una Educación REAL: Valores y Deporte". Este proyecto incluye varios programas de formación dirigidos a los entrenadores/as y deportistas (Fundación Realmadrid, 2012). Los padres y madres también están incluidos en este proyecto, dada la evidente importancia que tienen en la formación integral de sus hijos/as (Megías, 2010; Buceta y Beirán, 2002). Uno de los programas de este proyecto, denominado "La alineación de los Valores", ha tenido como objetivo elaborar dos cuadernos didácticos específicos: uno para el entrenador/ profesorado y otro para el jugador/alumnado. Se ha pretendido que estos materiales sirvan para orientar y ofrecer a los entrenadores/as los conocimientos y herramientas básicas que les ayuden a planificar e intervenir activamente. Asimismo, que favorezcan la evaluación continua tanto del profesorado como del colectivo que coordina dicho programa con el fin de recoger todas las experiencias, sugerencias y propuestas, de modo que permita la mejora de estos materiales y, por tanto, en el plan de la formación en valores educativos a través de la práctica deportiva (Ortega, Jiménez, Durán, Giménez, Jiménez, y Sáenz-López, 2010; Ortega, Jiménez, Durán, Giménez, Jiménez y Sáenz-López, 2011). Dichos cuadernos ofrecen múltiples propuestas, recomendaciones y dinámicas relacionadas con siete valores seleccionados para desarrollar este proyecto: Respeto, Autonomía, Motivación, Igualdad, Autoestima, Salud y Compañerismo. Se ha intentado que estos cuadernos sean un material de apoyo sencillo, atractivo y práctico, que permite a cada entrenador, según su nivel de formación y experiencia, decidir qué tipo de contenidos utilizar y cuándo es el momento oportuno. Asimismo, los jugadores/as pueden participar activamente construyendo sus propios aprendizajes en valores educativos a través de las diferentes propuestas de dinámicas y actividades. Ambos cuadernos se encuentran estrechamente interrelacionados.

El presente estudio ha tenido como finalidad los siguientes objetivos: a) utilizar los cuadernos didácticos como medio de apoyo para educar en valores a través de la práctica del baloncesto; b) conocer la percepción de los entrenadores/as respecto a la utilización de las diferentes propuestas y dinámicas del cuaderno; c) analizar los resultados obtenidos en el programa de valores educativos y llevar a cabo las propuestas de mejora tras la aplicación de estos materiales durante una temporada.

\section{Método}

En el presente estudio se ha utilizado una metodología basada en investigación-acción participativa (Carr y Kemmis, 1988); está enfocado hacia el fomento de valores sociales y personales, a través del baloncesto, de un colectivo de jóvenes y entrenadores/as pertenecientes a las Escuelas de Baloncesto de la Fundación Realmadrid. Los entrenadores/as implicados en el estudio en la temporada 2011-2012 han sido 17. Este trabajo ha sido realizado con 485 jugadores/as cuya distribución por categorías es la siguiente: babybasket (68), benjamín (97), alevín (108), infantil (121) y cadete (91).

Los materiales utilizados en esta investigación han sido a) los cuadernos didácticos del profesorado y del alumnado; b) cuestionario de evaluación del profesorado respecto a la utilización de los cuadernos didácticos; c) grupo de discusión; d) encuestas de satisfacción del programa de calidad de la Fundación Realmadrid.

Desde el inicio de la temporada los entrenadores/as han tenido a disposición los materiales didácticos y se les ha familiarizado para su utilización realizando unas recomendaciones para su aplicación en las escuelas. Además, se ha realizado un asesoramiento y seguimiento del trabajo realizado. Hacia la finalización de la temporada se les ha solicitado la elaboración de un cuestionario de evaluación y realizado un grupo de discusión.

\section{Resultados}

Con relación a la aplicación de los cuadernos didácticos, el grado de éxito subjetivo manifestado por los entrenadores/ as es elevado. Los valores más utilizados durante los entrenamientos han sido respeto, compañerismo, motivación y autonomía. El número de sesiones para trabajar cada valor, ha sido entre una y diez sesiones. La aplicación de estrategias de reflexión y comunicación, así como el establecimiento de normas concretas y ensalzar los comportamientos positivos, han sido los recursos más idóneos para mejorar los valores de los jugadores/as. Los entrenadores/as perciben que a los jugadores/as de categoría benjamín y alevín les gusta esta dinámica de trabajo. Destacan que hay algunos participantes que no les gusta reflexionar.

Respecto a los resultados del grupo de discusión en la fase final de la temporada, los entrenadores/as son conscientes de la necesidad de utilizar materiales didácticos para apoyar su labor educativa. A través de la puesta en marcha en la presente temporada, destacan algunas propuestas para tenerlas en consideración: a) se deben trabajar los valores educativos, siempre que sea posible, de forma simultánea con la actividad práctica de baloncesto; b) las tareas propuestas en los cuadernos han sido más receptivas en los jugadores/as de categorías benjamín y alevín; c) han descubierto que el fomento de la reflexión y el diálogo es positivo para mejorar el comportamiento de los jugadores/as; d) consideran que es importante que los jugadores/as no permanezcan mucho tiempo sentados mientras desarrollan alguna dinámica específica de un valor educativo; e) insisten en integrar el trabajo de valores junto a la mejora táctica y técnica de baloncesto; f) es preciso aplicar distintas dinámicas de trabajo según las diferentes categorías y edades de los jugadores/as; g) consideran que es bueno implicar a las familias y que estas valoran positivamente el 
trabajo realizado. La percepción de los entrenadores/as con relación a la participación de los padres y madres es positiva, sin embargo, algún entrenador manifiesta que cuando las familias han tenido que colaborar en el cuaderno de sus hijos/as, algunas han manifestado tener poco tiempo para estas tareas.

Los resultados de las encuestas de satisfacción realizada en la Fundación Realmadrid con 217 familias, de un total de 466, manifiestan los siguientes datos: a) el 84\% de las familias valoran como buena, o muy buena, la labor de las Escuelas de Baloncesto; b) el 93\% recomendaría a otras familias que llevaran a sus hijos/as a participar en estas escuelas; c) el respeto, el compañerismo y la autoestima son los valores más apreciados; d) los objetivos más destacados por las familias en la competición son: contribuir con el desarrollo integral de los jugadores/as; fomentar la diversión y mejorar a través de la competición todo lo aprendido en los entrenamientos; e) el 85\% considera que el modelo de competición de la Fundación Realmadrid fomenta la transmisión de valores educativos; f) entre las fortalezas atribuidas a las Escuelas de Baloncesto, destacan el trabajo realizado en valores educativos con un $35 \%$; a continuación la labor de los entrenadores/as con el $22 \%$; le sigue la motivación $17 \%$, la organización y la no competitividad, ambas con un $9 \%$.

\section{Discusión}

Los entrenadores/as de la Fundación Realmadrid son muy conscientes del valor educativo que puede conllevar la práctica del deporte (Gutiérrez, 2006; Jiménez, 2008; Jiménez y Durán, 2006). La filosofía y los objetivos de la Fundación Realmadrid planteados para el desarrollo de las Escuelas de Baloncesto son muy determinantes en este sentido y por esto se está llevando a cabo un amplio proyecto de educación en valores, al igual que otras fundaciones (FAD, 2009; Fundación Cultura y Deporte, 2012; Fundación FEB, 2012; Fundación Realmadrid 2012). Los resultados obtenidos en este estudio manifiestan que los entrenadores/as utilizan cuadernos didácticos, fundamentalmente para las categorías benjamín y alevín, que son edades comprendidas entre 8 y 12 años, y que coincide, en parte, con la franja de edad a la que va dirigido el programa de actuación en el deporte de la FAD (2009), así como el estudio realizado por Gutiérrez (2006). Destacamos también la necesidad de plantear tareas educativas específicas en función de las categorías, aspecto muy importante para evitar una generalización de propuestas en los materiales didácticos elaborados (Comunidad de Madrid, 2005).

El papel de las familias es relevante en el desarrollo del proyecto educativo planteado a través de la práctica del baloncesto y son determinantes en la contribución de valores de sus hijos/as (Megías, 2010). Los resultados de las encuestas plasman que existe una gran satisfacción del trabajo en valores realizado, y este aspecto favorece la labor llevada a cabo por los entrenadores/as y coordinadores/as de estas escuelas.

\section{Referencias}

Buceta, J. y Beirán, J. (2002) Los padres también juegan. Documento elaborado por el Gabinete de Psicología del Real Madrid.

Carr, W. y Kemmis, S. (1988). Teoría critica de la enseñanza. La investigación-acción en la formación del profesorado. Barcelona: Martínez Roca.

Comunidad de Madrid. (2005). Deporte, Olimpismo y Ciudadanía. Fichas pedagógicas. Madrid: Consejería de Cultura y Deportes de la Comunidad de Madrid.

Consejo Superior de Deportes (2004) Los valores del deporte en la educación. Más alto que uno mismo, Unidad didáctica, Consejo Superior de Deportes, Madrid.

FAD. (2009). Material didáctico del Club del Buen Deportista. Recuperado de http://www.fad.es/Publicaciones?id_nodo $=87$ హaccion $=0$ \& $t i p o=M D E$ okeyword $=$ Sauditoria $=F$

Fraile, A., Aguado, P., Díez, M., Fernández, M. Frutos, M., De Frutos, S. et al. (2008). Los conflictos en las clases de educación Física y algunas estrategias para su resolución. En A. Fraile (coord.) La resolución de los conflictos en y a través de la educación física. (pp. 7-64). Barcelona: Graó.

Fundación Cultura y Deporte de Castilla la Mancha. (2012). Programa Juego Limpio. Recuperado de http://www.fundacionculturaydeporte. com $/$ ? $q=$ node $/ 24 /$ print

Fundación FEB. (2012). Programas nacionales: Basket Integra. Recuperado de http://www.fundacion-feb.org/inicio.aspx?tabid=2

Fundación Realmadrid. (2012). Por una Educación REAL: Valores y Deporte. Realmadrid, (39), 42-47.

Gutiérrez, D. (2006). Modelo de intervención para educar en valores a través del fútbol: una experiencia con niños entre ocho y once ańos en el Colegio Mirabal. En Valores en movimiento. La actividad física y el

deporte como medio de educación en valores. (pp. 67-102). Madrid: Ministerio de Educación y Ciencia. CSD.

Hellison, D. (1995) Teaching personal and social responsability trough physical activity, Champaign, IL: Human Kinetics.

Jiménez, P.J. (2000) Modelo de intervención para educar en valores a jóvenes en riesgo a través de la actividad física y el deporte, Tesis doctoral, Universidad Politécnica de Madrid.

Jiménez, P.J. (2008). Manual de estrategias de intervención en actividad física, deporte y valores. Madrid: Síntesis.

Jiménez, P.J. y Durán. J. (Coords.). (2006): Valores en Movimiento: La Actividad física y el deporte como medio de educación en valores. Madrid: Ministerio de Educación y Ciencia. CSD.

Lleixá, T., y Soler, S. (2004). Experiencia y proyectos de actividad física y deportiva en entornos multiculturales. En T. Lleixá y S. Soler (coords.) Actividad física y deporte en sociedades multiculturales. ¿̇ntegración o segregación? (pp. 135-152). Barcelona: ICE. Universitat de Barcelona.

Megías, D. (2010). La importancia de la familia en la Educación Física. EF Deportes (143). Recuperado de http://www.efdeportes.com/efd143/ la-familia-en-la-educacion-fisica.htm

Mosquera, M. J. (2004) No violencia en el deporte y en la vida, Guía para docentes, Xunta de Galicia, A Coruña.

Ortega, G., Jiménez, P.J., Durán, L.J., Giménez, F.J., Jiménez, A.C., y Sáenz-López, P. (2010). Por una Educación REAL. Valores y Deporte. La Alineación de los Valores. Cuaderno del Profesorado. Madrid: Ediciones Gráficas Fundación Realmadrid.

Ortega, G., Jiménez, A.C., Durán, L.J., Giménez, F.J., Jiménez, P.J., y Sáenz-López, P. (2011). Por una Educación REAL. Valores y Deporte. La 
Alineación de los Valores. Cuaderno del Alumnado. Madrid: Ediciones

Gráficas Fundación Realmadrid. 